\title{
Un numéro commun SFP-UdPPC sur la physique des matériaux anciens
}

En 2010 et 2013, la Société Française de Physique et l'Union des Professeurs de Physique et de Chimie avaient uni leurs forces éditoriales pour publier conjointement des numéros spéciaux de la revue Reflets de la physique et du Bulletin des Professeurs de Physique et de Chimie sur le laser, puis sur le rayonnement synchrotron. Ce nouveau numéro spécial commun veut illustrer la complémentarité de la physique moderne et de l'histoire de l'art.

Jusqu'au $18^{\mathrm{e}}$ siècle, de grands savants avaient une vue d'ensemble des connaissances humaines en arts et en sciences. Mais, à partir du $19^{\mathrm{e}}$ siècle et surtout au $20^{\mathrm{e}}$ siècle, l'explosion des connaissances dans tous les domaines (physiques relativiste et quantique, code génétique, informatique..., mais aussi sociologie, psychologie et économie) ont amené inévitablement une spécialisation de plus en plus grande des chercheurs et des enseignants. Et la complexité croissante des sciences dures a conduit une grande partie du public et surtout des jeunes à s'en détourner. Or, ces deux mondes, les arts et les sciences, connaissent des interactions fortes qu'il est nécessaire de rétablir.

C'est dans cette optique que se situe ce numéro spécial, qui vise à montrer que la physique n'est pas un domaine clos, mais apporte considérablement à d'autres disciplines, ici l'étude des matériaux anciens (peintures, sculptures, céramiques, objets préhistoriques, bâtiments...) : datation, méthodes de fabrication ou de construction, propriétés optiques, vieillissement et restauration... Les disciplines impliquées de la physique sont multiples : optique et physique des lasers, spectroscopie, physique nucléaire, science des matériaux, simulations numériques...

En retour, l'étude des matériaux anciens apporte également à la physique : elle la fait passer de l'étude de systèmes relativement simples à celle d'objets très complexes et hétérogènes, obligeant à développer des techniques physiques et des programmes numériques adaptés.

Il est particulièrement important de montrer au grand public, et encore plus aux enseignants et aux élèves, que la physique irradie la vie de tous les jours et les autres matières, en particulier celles qui séduisent par leur mystère et leur beauté.

Nous espérons que ce numéro spécial commun - qui témoigne également des relations étroites et historiques entre la Société Française de Physique et I'Union des Professeurs de Physique et de Chimie - sera pour vous l'occasion de belles découvertes.

Catherine Langlais

Présidente de la Société Française de Physique

Marie-Thérèse Lehoucq

Présidente de l'Union des Professeurs de Physique et de Chimie 